\title{
Micro-RNAs and Their Roles in Breast Cancer Pathogenesis; An Updated Review Article
}

\author{
Mohammad Hossein Bakhshi Aliabad', Shahnaz Kheirandish¹, Zeynab Zarezade1, Nasim Saeidi', Hamid \\ Kabiri ${ }^{1}$, Zahra Khazaei ${ }^{1}$, Hossein Sahamifar ${ }^{1}$, Fatemeh Taghvaei ${ }^{1}$, Sepideh Jafarzadeh${ }^{1}$, Masoud Zare ${ }^{1}$, Sajad

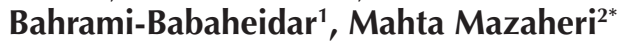 \\ ${ }^{1}$ MSc, Student of Genetics, Faculty of Medecine, Shahid Sadoughi University of Medical Sciences, International Campus, \\ Yazd, Iran \\ ${ }^{2}$ Associate Professor of Medical Genetics, Department of Genetics, Faculty of Medicine, Shahid Sadoughi University of \\ Medical Sciences, Yazd, Iran
}

*Correspondence to

Mahta Mazaheri, MD, PhD;

Email: m.mazaheri@ssu.ac.ir

Received August 9, 2016 Revised August 17, 2016 Accepted August 21, 2016 Published online September 20, 2016

Please cite this article as follows: Bakhshi Aliabad MH, Kheirandish S, Zarezade Z, et al. Micro-RNAs and their roles in breast cancer pathogenesis; an updated review article. Int J Basic Sci Med. 2016;1(2):4852. doi:10.15171/ ijbms.2016.11.

\begin{abstract}
Micro-RNAs (miRNAs) are small molecules that modulate the translation of target mRNA through binding to these molecules. In cancer, the expression of oncogenes and suppressors are increased and decreased, respectively. MiRNAs are one of the causing factors in the formation of cancer. To collect data, research articles in PubMed and Google Scholar were searched under keywords including breast cancer and micro-RNA. The data obtained from articles published in prestigious journals showed that some miRNAs are involved in the suppression and some in the induction of cancer cell growth in breast cancer tissue. Some of them are also involved in the aggression and metastasis. According to the obtained results, it seems that assessing miRNAs in breast cancer tissue can be considered as a potential candidate for prognosis and may be used in pre-diagnosis of the disease in near future.

Keywords: Breast cancer, Micro-RNA, Pathogenesis.
\end{abstract}

\section{Background and Objectives}

Micro-RNAs (miRNAs) were discovered in 1993 by Rosalind Lee and Rhonda Feinbaum while working on the lin-14 gene in the C. Elegans. ${ }^{1}$ In fact, one of the most important recent advances in biochemical research was the discovery of noncoding long 22 nucleotide RNAs called miRNAs that are involved in regulating genes' expression, ${ }^{2}$ via mRNA degradation or preventing its translation and modulating a variety of basic cellular processes. ${ }^{3}$ Today a few number of miRNAs have been studied and the performance of this number included cell differentiation, proliferation, apoptosis, and anti-viral and anti-cancer defenses. ${ }^{5}$ About 50\% of miRNAs are placed within genes and it is expected that approximately $50 \%$ of them be placed in introns. More than half of miRNA genes are located on chromosome's fragile sites that allow duplication, deletion, and cell movement during the cancer development process. These areas can affect the expression of miRNAs. Recent research has shown that these molecules can act as either onco- genes or tumor suppressors. ${ }^{6}$ Given the importance of etiology and treatment of tumors and due to many unknown causes of cancers, ${ }^{7}$ extensive studies have discussed around the role of these micro molecules in the pathogenesis of cancers. Many studies have examined the possible molecules involved in cancer. ${ }^{9,10}$ Today, several review articles have been published regarding several aspects of miRNAs in breast cancer. In this review article, we attempted to collate recent articles regarding miRNAs in breast cancer to update our knowledge and discuss about the production and performance of miRNAs and their involvement in the pathogenesis of breast cancer.

\section{Biogenesis of Micro-RNA}

Biogenesis and production of these molecules begin from the nucleus and continues to the cytoplasm.

Production Pathway of Micro-RNA in the Nucleus

In nucleus, the miRNA genes are transcribed by RNA polymerase II. ${ }^{11}$ In the

Copyright $\odot 2016$ The Author(s); Published by Zabol University of Medical Sciences. This is an open-access article distributed under the terms of the Creative Commons Attribution License (http://creativecommons.org/licenses/by/4.0), which permits unrestricted use, distribution, and reproduction in any medium, provided the original work is properly cited. 
next step, RNA is looped which is called pri-miRNA. ${ }^{11}$ Single-stranded RNA in upstream and downstream of miRNA is essential for the action of Drosha enzyme (nuclear RNase III) in the core. Drosha enzyme cuts and changes precursor of the miRNAs into pre-miRNAs by interaction with nuclear proteins called DiGeorge syndrome critical region. ${ }^{12}$ MiRNA precursor has 65 nucleotides and is transferred by Exportin- 5 proteins to the cytoplasm. ${ }^{13}$

\section{Micro-RNA Biogenesis Pathway in the Cytoplasm}

The Dicer enzyme (ribonuclease type III) in the cytoplasm cuts 22 nucleotides from the $3^{\prime}$ end and makes mature miRNA. ${ }^{11,14}$

\section{Performance of RNA-Induced Silencing Complex}

The complex actually consists of miRNA and nucleoprotein, that is why it is called RISC (RNA-induced silencing complex). ${ }^{15}$ In addition to the miRNAs, it also consists of a family of Argonauts proteins. ${ }^{15}$ During the function of miRNA, the molecule must be set to RISC complex to produce a leader RNA and a transient RNA that normally degrade. ${ }^{15}$ The resulting molecules are directed to target RNAs that contain complementary sequences with guide RNA. ${ }^{15}$ Target RNA is either degraded or its translation is disrupted. In cases where the target RNA degrades, Argonauts that is a subunit of this complex cuts the initial mRNA. ${ }^{15}$ This protein has subunits bound to the target RNA and is the RNA subunit cutter (ribonuclease $\mathrm{H}$ ). The second target RNA-binder selects the $3^{\prime}$ end, and a second cutter that should cut is placed in the vicinity of RNA. ${ }^{15}$ Cutting the middle region of double-stranded target mRNA as well as guide and intermediate RNA is performed from the $5^{\prime}$ end. ${ }^{13}$

In fact, the main function of miRNAs is regulating the expression of genes. ${ }^{16} \mathrm{MiRNA}$ is the complement of part of one or more target RNA. ${ }^{17}$ Creation of base pair in promoter region causes cuts in target RNA and prevents its translation and controls it. Sometimes the pairing amount is very low, about 2 to 7 base pairs. ${ }^{17}$ In such cases, energy of miRNAs is less, while, more pairing leads to higher energy of the miRNA. ${ }^{17}$ MiRNAs would render their influence on cells by affecting gene methylation and histone modifications and several additional methods on the gene expression. ${ }^{18}$

\section{Micro-RNA Actions in Cell Biology and Diseases}

These molecules act in various biochemical and immunologic pathways. Apoptosis is a main pathway which is affected by this molecule. MiRNAs play an important role in the process of homeostasis and cancer development by regulating this pathway. ${ }^{19}$ Numerous studies have focused on the role of miRNAs in the cell biogenesis and their immortality and therefore cancer formation. ${ }^{20}$ Bioinformatics and micro-array studies have shown that migration inhibitory factor (MIF), the oncogenic factor causing the inhibition of macrophage migration, is the target of miRNA-451 (miR-451). ${ }^{21}$ Overexpression of these molecules would result in reduced gene expression and MIF protein synthesis, and thereby reducing the expression of target gene receptor. ${ }^{22}$ In another study aimed to investigate the molecular mechanism of miR-192 on colon cancer, cell lines of human colon cancer with different modes of P53 were studied and the effect of these miRNA on cell proliferation, cell cycle, and mechanisms regulating gene expression were assessed. The results showed that one of the target genes of miR-192 was dihydrofolate reductase. This molecule targeted cell proliferation using the p53-miRNA cycle. ${ }^{23,24}$ Besides colon cancer, the impact of miRNAs in different cancers including unusual expression of miRNAs in leukemia was studied. ${ }^{25}$ Another study indicated that changes in gene expression of some miRNAs are clearly visible in breast cancer and healthy cells; so, cancerous cells can be differentiated by measuring their gene expression. Thus, it appears that miRNAs play key roles in the pathogenesis and in the mechanisms leading to the development of cancers. We further discuss the role of miRNAs in the pathogenesis and diagnosis of breast cancer.

\section{Methods}

In order to find recent investigations regarding the roles of miRNAs in breast cancer, three main databases including PubMed, Scopus, and Google Scholar were searched using "miRNA, micro-RNA, and breast cancer" key words.

\section{The Role of Micro-RNA in Breast Cancer}

MiRNAs are one of the molecular factors affecting breast cancer. ${ }^{26}$ Inhibitor miRNAs suppress cancer-stimulating genes, and principally the expression of these miRNAs in breast cancer is reduced ${ }^{26}$ On the contrary, specific miRNAs stimulate breast cancer development. ${ }^{26}$ Inhibition and reduction of regulation in the oncogenic RNAs may play an important role in the treatment of breast cancer, while increased expression of inhibitor miRNAs can have anticancer therapeutic effects. ${ }^{27}$ Therefore, the reductions in the expression of some specific miRNAs in breast cancer suggest that the miRNAs are responsible for cell division and apoptosis in the cell system. ${ }^{27}$

\section{Reduced Micro-RNAs in Breast Cancer}

Some studies have shown that miR-206 is significantly reduced in the breast cancer patients with positive alpha-estrogen receptor. ${ }^{28}$ The studies also show that mRNA of the oncogenes is the target of two miRNAs including miR-125 and miR-145, and is destroyed/degraded under the effect of these two molecules and therefore the proliferation of cells through this pathway is prevented. ${ }^{29}$ Of the oncogenes targeted by these two miRNAs are YES proto-oncogene and $C B F B$ (core-binding factor subunit beta) transcription factor. ${ }^{29}$ Interestingly, the decreased expression of these two miRNAs is reported in breast cancer tissue. ${ }^{29}$ The study by Foekens and colleagues showed that the expression level of miR-210 is significantly reduced in breast cancer tissue. ${ }^{30}$ Decreased expression of miR-27b was reported in another study in 2006 by Tsuchiya et al, conducted on a specific population of patients with breast 
cancer in Japan. ${ }^{31}$ Let-7 is another micro-RNA with reduced expression in breast cancer and the decrease in the expression of this molecule is associated with poor prognosis in breast cancer that is combined with increased expression of CYP1B1 (cytochrome P450 1B1) oncogene. ${ }^{32}$ On the other hand, investigations show that some mutations in miR-196a2 and miR-499, which lead to the dysfunction of these molecules, are associated with the increased risk of breast cancer. ${ }^{33}$ Studies on the miR-200 family (miR-200a, miR-200b, and miR-200c), miR-141, miR-429, and miR-205 showed a significant reduction in the expression of these molecules in cancer cells. ${ }^{33}$ Recent studies suggest that miR-17-5p/miR-20a inhibits cell proliferation with negative regulation of cyclin D1, and the decreased expression of these molecules is associated with breast cancer. ${ }^{34}$ Moreover, the inhibitory role of miR-31 on metastasis in breast cancer has been demonstrated in previous studies. $^{7}$

According to the results achieved in the presented studies, it seems that miRNAs b27, 31, 125, 141, 145, a2196, 200 family, 205, 206, 210, 429, and 499 along with let-7 are the molecules that have pre-apoptotic function and reduce the expression of oncogenic proteins and increase the expression of anti-apoptotic molecules. Accordingly, it may be concluded that the prevention of cancers such as breast cancer is plausible by increasing their expression.

\section{Oncogene Micro-RNAs in Breast Cancer}

Some miRNAs, unlike previous cases, not only have no pre-apoptotic role, but act as an anti-apoptotic molecule, because they play an important role through targeting the molecules involved in apoptosis and creation of immortal cells. ${ }^{7}$ Interestingly, these micro-molecules increase sharply in breast cancer and in addition to a vital role in making cells immortal, they are involved in metastasis and exacerbating the disease. ${ }^{7}$ These miRNAs can be targeted in molecular therapies in breast cancer. ${ }^{7}$ For example, studies have shown that miR-21 is an oncogene in tumorigenesis, metastasis, and aggressiveness, and is associated with poor prognosis in breast cancer. Interestingly, miR-21 target genes are all tumor suppressor (phosphatidylinositol-3,4,5-trisphosphate 3-phosphatase $[P T E N]$, tropomyosin1, programmed cell death protein 4 [PDCD4] $)^{35}$ These micromolecules target the cell cycle regulatory proteins such as PTEN and CDC25 (cell division cycle 25), and play an important role in the cell proliferation and metastasis. ${ }^{33}$ Increased expression of miR-155 and miR-9-1 in breast cancer indicate the oncogenicity of these molecules. ${ }^{36}$

\section{Micro-RNAs and Metastasis in Breast Cancer}

As abovementioned, some miRNAs are associated with metastasis and malignant breast cancer. Based on previous studies, it seems that some miRNAs reinforce the role of metastatic features in breast cancer, like miR-301, miR-103/107, miR-21, miR-9, miR181b-1, miR-17/92, miR-489, miR-495, miR-200c, and miR-373. ${ }^{37,38}$ Some miRNAs are also recognized to play a role in the devel-

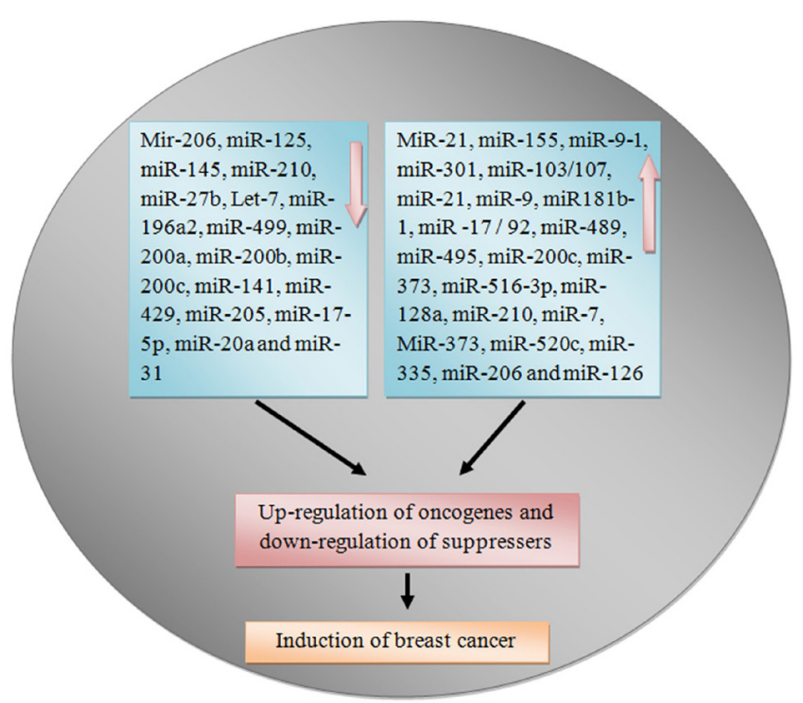

Figure 1. The most important miRNAs involved in the induction of malignancy in the breast cells. Figure illustrates that reduced and increased expression of anti-oncogenic and anti-suppresser miRNAs leads to the up-regulation of oncogenes and also downregulation of suppressers which results in the induction of breast cancer.

opment of aggressive and progressive form of breast cancer, like miR-516-3p, miR-128a, miR-210, and miR-7.. ${ }^{39}$ MiR-373 and miR-520c stimulate cancer cell migration and aggressiveness. ${ }^{40}$ In addition, decreased expression of miR-335, miR-206, and miR-126 in the metastatic tumor cells represent the anti-cancer effects of these miRNAs (Figure 1). ${ }^{41}$

\section{Conclusion}

Given the role of miRNAs in cancer induction or prevention as well as invasiveness or non-invasiveness, it seems that these micro molecules can be used as biomarkers for early detection of breast cancer and progression. Release of miRNAs from the tumor to the blood circulation is performed through the exosome vesicles and apoptotic bodies, hence, miRNAs present in blood circulation can be used as diagnostic indicators. ${ }^{42}$ Therefore, the levels of miRNAs' expression can be considered in the monitoring of tumor status. It is worth to note that the removal of primary tumor reduces the rotational miRNAs. Hence, this investigation showed that we can consider these micro molecules in the identification of patients' status and treatment pathways.

\section{Authors Contributions}

MHBA has designed the project and assembled the collected data and has prepared the manuscript for submission. SK, ZZ, NS, HK, ZK, HS, FT, SJ, MZ, and SB-B have collected the data. MM has written the $80 \%$ of the article. The authors have approved the final version of the manuscript.

\section{Ethical Approval \\ Not applicable.}




\section{Competing Interests}

Authors declare that they have no competing interests.

\section{Acknowledgments}

This study was supported by Yazd University of Medical Sciences and all rights are reserved for the University's research deputy. The authors of this manuscript are grateful to all those who cooperated in this project.

\section{References}

1. Lee RC, Feinbaum RL, Ambros V. The C. elegans heterochronic gene lin-4 encodes small RNAs with antisense complementarity to lin-14. Cell. 1993;75(5):843854. doi:10.1016/0092-8674(93)90529-y.

2. Kriegel AJ, Baker MA, Liu Y, Liu P, Cowley AW, Liang M. Endogenous microRNAs in human microvascular endothelial cells regulate mRNAs encoded by hypertension-related genes. Hypertension. 2015;66(4):793799. doi:10.1161/hypertensionaha.115.05645.

3. Barnes CJ, Li F, Mandal M, Yang Z, Sahin AA, Kumar R. Heregulin induces expression, ATPase activity, and nuclear localization of G3BP, a Ras signaling component, in human breast tumors. Cancer Res. 2002;62(5):1251-1255.

4. Domingo E, Holland J. RNA virus mutations and fitness for survival. Ann Rev Microbiol. 1997;51(1):151-178. doi:10.1161/hypertensionaha.115.05645.

5. Ambros V. The evolution of our thinking about microRNAs. Nature Med. 2008;14(10):1036-1040. doi:10.1038/nm10081036.

6. Shenouda SK, Alahari SK. MicroRNA function in cancer: oncogene or a tumor suppressor? Cancer Metastasis Rev. 2009;28(3-4):369-378. doi:10.1007/s10555-009-9188-5.

7. Motamedi S, Majidzadeh K, Mazaheri M, Anbiaie R, Mortazavizadeh SM, Esmaeili R. Tamoxifen resistance and CYP2D6 copy numbers in breast cancer patients. Asian Pac J Cancer Prev. 2012;13(12):6101-6104.

8. Mazaheri Naeeini M, Kochanova A, Majidzadeh AK, Richard-Foy H, Bystricky K. Molecular and cellular basis of anti-estrogen behavior in breast cancer cells. Eur J Cancer Suppl. 2010;8(3):40-44. doi:10.1016/s1359-6349(10)700859.

9. Mazaheri Naeeini M, Hoseinzadeh L, Motaleb G, Majidzadeh K. HER2 methylation changes in breast cancer patients. Rostamineh 2015;4(6):16-25.

10. Motalleb G, Pourrahmat E, Rashki A, et al. Epidermal growth factor receptor gene expression evaluation in colorectal cancer patients. Indian J Cancer. 2014;51(3):358. doi:10.4103/0019-509x.146771.

11. Bartel DP. MicroRNAs: genomics, biogenesis, mechanism, and function. Cell. 2004;116(2):281-297.

12. Kim VN. MicroRNA biogenesis: coordinated cropping and dicing. Nature Rev Mol Cell Biol. 2005;6(5):376-385.

13. Gregory RI, Chendrimada TP, Cooch N, Shiekhattar R. Human RISC couples microRNA biogenesis and posttranscriptional gene silencing. Cell. 2005;123(4):631640. doi:10.1016/j.cell.2005.10.022.

14. Deng S, Calin GA, Croce CM, Coukos G, Zhang L. Mechanisms of microRNA deregulation in human cancer. Cell Cycle. 2008;7(17):2643-2646. doi:10.4161/ cc.7.17.6597.

15. Yang L, Zheng LY, Tian Y, et al. C6 ceramide dramatically enhances docetaxel-induced growth inhibition and apoptosis in cultured breast cancer cells: a mechanism study. Exp Cell Res. 2015;332(1):47-59. doi:10.1016/j. yexcr.2014.12.017.

16. Motalleb G, Pourrahmat E, Najafi S, et al. Epidermal growth factor receptor gene expression evaluation in colorectal cancer patients. Indian J Cancer 2014;51(2):358362 . doi:10.1016/j.yexcr.2014.12.017.

17. Lu LF, Liston A. MicroRNA in the immune system, microRNA as an immune system. Immunology 2009;127(3):291-8. doi:10.1111/j.1365-2567.2009.03092.x.

18. Pillai RS, Bhattacharyya SN, Artus CG, et al. Inhibition of translational initiation by Let-7 MicroRNA in human cells. Science. 2005;309(5740):1573-1576.

19. Fisher PB, Gopalkrishnan RV, Chada S, et al. mda-7/IL24, a novel cancer selective apoptosis inducing cytokine gene: from the laboratory into the clinic. Cancer Biol Ther. 2003;2:22-36. doi:10.4161/cbt.458.

20. Katada $\mathrm{T}$, Ishiguro $\mathrm{H}$, Kuwabara $\mathrm{Y}$, et al. MicroRNA expression profile in undifferentiated gastric cancer. Int J Oncol. 2009;34(2):537-542.

21. Kocanova S, Mazaheri M, Caze-Subra S, Bystricky K. Ligands specify estrogen receptor alpha nuclear localization and degradation. BMC Cell Biol. 2010;11(1):98.

22. Godlewski J, Bronisz A, Nowicki MO, Chiocca EA, Lawler S. MicroRNA-451: A conditional switch controlling glioma cell proliferation and migration. Cell Cycle. 2010;9(14):2814-2820. doi:10.4161/cc.9.14.12248.

23. Braun CJ, Zhang X, Savelyeva I, et al. p53-Responsive micrornas 192 and 215 are capable of inducing cell cycle arrest. Cancer Res. 2008;68(24):10094-10104. doi:10.1158/0008-5472.can-08-1569.

24. Song B, Wang Y, Kudo K, Gavin EJ, Xi Y, Ju J. miR-192 Regulates dihydrofolate reductase and cellular proliferation through the p53-microRNA circuit. Cli Cancer Res. 2008;14(24):8080-8086. doi:10.1158/1078-0432.ccr-081422.

25. Zanette D, Rivadavia F, Molfetta G, et al. miRNA expression profiles in chronic lymphocytic and acute lymphocytic leukemia. Braz J Med Biol Res. 2007;40(11):1435-1440.

26. Shi M, Guo N. MicroRNA expression and its implications for the diagnosis and therapeutic strategies of breast cancer. Cancer Treat Rev. 2009;35(4):328-34. doi:10.1016/j. ctrv.2008.12.002.

27. Kota SK, Balasubramanian S. Cancer therapy via modulation of micro RNA levels: a promising future. Drug discovery today 2010;15(17):733-40. doi:10.1016/j. drudis.2010.07.003.

28. Kondo N, Toyama T, Sugiura H, Fujii Y, Yamashita H. miR-206 Expression Is Down-regulated in Estrogen Receptor a-Positive Human Breast Cancer. Cancer Res. 2008;68(13):5004-5008. doi:10.1158/0008-5472.can-080180 .

29. Johnson SM, Grosshans H, Shingara J, et al. RAS is regulated by the let-7 microRNA family. Cell. 2005;120(5):635-647. doi:10.1016/j.cell.2005.01.014.

30. Wu F, Zikusoka M, Trindade A, et al. MicroRNAs are differentially expressed in ulcerative colitis and alter expression of macrophage inflammatory peptide-2a. Gastroenterology. 2008;135(5):1624-35.e24. doi:10.1053/j. gastro.2008.07.068.

31. Tsuchiya Y, Nakajima M, Takagi S, Taniya T, Yokoi T. MicroRNA regulates the expression of human cytochrome P450 1B1. Cancer Res. 2006;66(18):9090-9998. 
doi:10.1158/0008-5472.CAN-06-1403.

32. $\mathrm{Hu} \mathrm{Z}$, Liang J, Wang $\mathrm{Z}$, et al. Common genetic variants in pre-microRNAs were associated with increased risk of breast cancer in Chinese women. Hum Mutat. 2009;30(1):79-84.

33. Kaboli PJ, Rahmat A, Ismail P, Ling KH. Targets and mechanisms of berberine, a natural drug with potential to treat cancer with special focus on breast cancer. Eur J Pharmacol. 2014;740:584-595.

34. Valastyan S, Reinhardt F, Benaich N, et al. A pleiotropically acting microRNA, miR-31, inhibits breast cancer metastasis. Cell. 2009;137(6):1032-1046. doi:10.1016/j. cell.2009.03.047.

35. Qian B, Katsaros D, Lu L, et al. High miR-21 expression in breast cancer associated with poor disease-free survival in early stage disease and high TGF- $\beta 1$. Breast Cancer Res Treat. 2009;117(1):131-40. doi:10.1007/s10549-008-02197.

36. Volinia S, Calin GA, Liu CG, et al. A microRNA expression signature of human solid tumors defines cancer gene targets. PNAS. 2006;103(7):2257-61. doi:10.1073/ pnas.0510565103.
37. Ma L, Reinhardt F, Pan E, et al. Therapeutic silencing of miR-10b inhibits metastasis in a mouse mammary tumor model. Nature Biotechnol. 2010;28(4):341-347. doi:10.1038/nbt.1618.

38. YahyaSM,ElsayedGH.A summary for molecular regulations of miRNAs in breast cancer. Clin Biochem. 2015;48(6):388396. doi:10.1016/j.clinbiochem.2014.12.013.

39. Foekens JA, Sieuwerts AM, Smid M, et al. Four miRNAs associated with aggressiveness of lymph node-negative, estrogen receptor-positive human breast cancer. PNAS. 2008;105(35):13021-6. doi:10.1073/pnas.0803304105.

40. Huang Q, Gumireddy K, Schrier M, et al. The microRNAs miR-373 and miR-520c promote tumour invasion and metastasis. Nature Cell Biol. 2008;10(2):202-210. doi:10.1038/ncb1681.

41. Tavazoie SF, Alarcón C, Oskarsson T, et al. Endogenous human microRNAs that suppress breast cancer metastasis. Nature. 2008;451(7175):147-152. doi:10.1038/nature06487.

42. Iguchi $\mathrm{H}$, Kosaka N, Ochiya T. Secretory microRNAs as a versatile communication tool. Commun Integr Biol. 2010;3(5):478-81. doi:10.4161/cib.3.5.12693. 